\title{
FUNDAMENTAL PLANE AND MERGER SCENARIO
}

\author{
K. BEKKI \\ Astronomical Institute, Tohoku University, Sendai, 980-77, \\ Japan
}

\section{Introduction}

The Fundamental Plane (FP) is one of the most important universal relations in early type galaxies because it contains valuable information about the formative and evolutionary process of galaxies (Djorgovski \& Davis 1987, Dressler et al. 1987). The commonly used form of the scaling relation in the FP is described as $R_{\mathrm{e}}=\sigma^{A} I^{B}$, where $R_{\mathrm{e}}, \sigma$, and $I$ are effective radius, central velocity dispersion, and mean surface brightness of elliptical galaxies, respectively. The exponents $A, B$ are considered to be $1.56 \pm 0.07$ and $-0.94 \pm 0.09$ in the FP derived by $K$ band photometry, respectively, and these values deviate significantly from the values $A=2.0$ and $B=-1.0$ expected from virial theorem (Pahre et al. 1995; Djorgovski, Pahre, \& de Carvalho 1996). This apparent deviation requires that the ratio of dynamical mass $(M)$ to luminosity of elliptical galaxies $(L)$ depends on $M$ as $M / L$ $\propto M^{\alpha}(\alpha=0.12 \pm 0.03$ for $K$ band). Possible interpretations for the required dependence of $M / L$ on $M$ are generally considered to be divided into the following two. One is that the required dependence of $M / L$ on $M$ results from the fact that the mean stellar age and metalicity of elliptical galaxies depend systematically on $M$. The other is that the required dependence reflects the $M$ dependence of structural and kinematical properties of elliptical galaxies ("nonhomology"). Although we should not neglect the importance of stellar populations in generating the $M$ dependence of the $M / L$ (Renzini \& Ciotti 1993), we here consider that the origin of the required $M$ dependence of $M / L$ is closely associated with the structural and kinematical properties dependent on $M$ or $L$ in elliptical galaxies.

The purpose of this contribution paper is to elucidate the origin of the luminosity-dependent structural and kinematical properties ("nonhomology") in elliptical galaxies and thereby to explore the physical meaning of the FP. We here focus particularly on the star formation history of elliptical 
galaxies and accordingly investigate the important roles of the star formation history in generating the structural and kinematical nonhomology of elliptical galaxies. In investigating the non-homologous nature in elliptical galaxies, we adopt the "merger hypothesis" in which elliptical galaxies are formed by galaxy mergers between two late-type spirals (Toomre \& Toomre 1977). In particular, we consider galaxy mergers between two disk galaxies with a gas mass fraction larger than 0.2 observed in the present typical late-type spirals in order to mimic elliptical galaxy formation by galaxy mergers at higher redshift.

\section{Model}

We construct models of galaxy mergers between star-forming gas-rich disk galaxies with equal mass by using Fall-Efstathiou model (1980). The details of the model are given by Bekki \& Shioya (1997). We adopt the Schmidt law (Schmidt 1959) with exponent $\gamma$ as the controlling parameter of the rate of star formation. The $\gamma$ is set to be 2.0 for all the simulations in the present study. The amount of gas consumed by star formation for each gas particle in each time step, $\dot{M}_{\mathrm{g}}$, is given as:

$$
\dot{M}_{\mathrm{g}} \propto C_{\mathrm{SF}} \times\left(\rho_{\mathrm{g}} / \rho_{0}\right)^{\gamma-1.0}
$$

where $\rho_{\mathrm{g}}$ and $\rho_{0}$ are the gas density around each gas particle and the mean gas density at 0.48 radius of an initial disk, respectively. The $C_{\mathrm{SF}}$ in the equation (1) is the parameter that controls the rapidity of gas consumption by star formation: The larger the $C_{\mathrm{SF}}$ is, the more rapidly the gas particles are converted to new stellar particles.

We here investigate fundamental roles of the rapidity of star formation $\left(C_{\mathrm{SF}}\right)$ in determining structural and kinematical properties of merger remnants. To be specific, we investigate the $C_{\mathrm{SF}}$ dependence of the following three nonhomology parameters: $k_{\mathrm{S}} \propto R_{\mathrm{e}} / R_{\mathrm{g}}, k_{\mathrm{K}} \propto\left(1.0+c_{\mathrm{v}} \times\left(v_{\mathrm{m}} / \sigma_{0}\right)^{2}\right)$, $k_{\mathrm{M}} \propto M_{\mathrm{t}} / L \propto M_{\mathrm{t}} / M_{\mathrm{b}}$. In the above equations, the $R_{\mathrm{e}}, R_{\mathrm{g}}, v_{\mathrm{m}}, \sigma_{0}, M_{\mathrm{t}}, M_{\mathrm{b}}$, and $L$ represent effective radius, gravitational radius, maximum rotational velocity, central velocity dispersion, total mass, total baryonic mass, and luminosity of a merger remnant, respectively. The value of the parameter $c_{\mathrm{v}}$ depends on the details of radial distribution of kinematical properties of galaxies. In the present study, we adopt 0.81 for the $c_{\mathrm{v}}$, which is the same as that of Prugniel \& Simien (1996). These three nonhomology parameters must cooperate to satisfy the following relation inferred from the FP.

$$
K_{\mathrm{FP}} \propto k_{\mathrm{S}} k_{\mathrm{K}}{ }^{-1} k_{\mathrm{M}} \propto \sigma_{0}^{2} / R_{\mathrm{e}} / I_{\mathrm{e}} \propto M / L \propto L^{\alpha}
$$

where $I_{\mathrm{e}}$ is mean surface luminosity of elliptical galaxies. The $K_{\mathrm{FP}}$ represents "total" nonhomology of galaxies, which includes the above three different types of nonhomology. If elliptical galaxies do not have non-homologous 
nature, the $K_{\mathrm{FP}}$, which corresponds to the conventionally used $M / L$, is constant for all elliptical galaxies.

\section{Results}

Fig. 1 describes the $C_{\mathrm{SF}}(=7.0,3.5,1,75,0.7$, and 0.35$)$ dependence of the nonhomology parameters, $k_{\mathrm{S}}, k_{\mathrm{K}}$, and $k_{\mathrm{M}}$ in the merger remnants. In order to show more clearly characteristics of the $C_{\mathrm{SF}}$ dependence of $k_{\mathrm{S}}, k_{\mathrm{K}}$, and $k_{\mathrm{M}}$, we preset the best fitted line derived by least square fitting of each set of experimental data to assumed relations like as $k_{\mathrm{S}}\left(k_{\mathrm{K}}\right.$ and $\left.k_{\mathrm{M}}\right) \propto C_{\mathrm{SF}}{ }^{x}$, where the exponent $x$ is described below. We can observe clear trends in the $C_{\mathrm{SF}}$ dependence of $k_{\mathrm{S}}, k_{\mathrm{K}}$, and $k_{\mathrm{M}}$ as follows. First, as is shown in the left panel of Fig. $1, k_{\mathrm{S}}$ is appreciably larger for models with larger $C_{\mathrm{SF}}$. The reason for this dependence is that the rapidity of star formation of mergers basically determines how much amount of stellar component is transferred to the central region of the remnants during mergers, which is a key factor for final mass distribution of stellar component in merger remnants. The dependence of $k_{\mathrm{S}}$ on $C_{\mathrm{SF}}$ is described as $k_{\mathrm{S}} \propto C_{\mathrm{SF}}{ }^{0.25}$. Second, we can observe in the middle panel of Fig. 1 that the larger the $C_{\mathrm{SF}}$ is, the smaller the $k_{\mathrm{K}}$ is. This is because total amount of gaseous dissipation during merging, which is smaller for models with larger $C_{\mathrm{SF}}$, principally determines how efficiently initial total potential energy of a galaxy merger can be converted into rotational energy rather than random kinetic energy during galaxy merging and thus how strongly the merger remnant is dynamically supported by global rotation. The dependence of $k_{\mathrm{K}}$ on $C_{\mathrm{SF}}$ is described as $k_{\mathrm{K}} \propto C_{\mathrm{SF}}{ }^{-0.08}$. Third, $k_{\mathrm{M}}$ is larger for models with larger $C_{\mathrm{SF}}$. This is because in the model with larger $C_{\mathrm{SF}}$, less amount of stellar component is transferred to the central region owing to less amount of kinetic energy dissipated away by gaseous dissipation during merging. The dependence of $k_{\mathrm{M}}$ on $C_{\mathrm{SF}}$ is described as $k_{\mathrm{M}} \propto C_{\mathrm{SF}}{ }^{0.11}$. Thus, the dependence of $K_{\mathrm{FP}}$ on $C_{\mathrm{SF}}$ is described as $K_{\mathrm{FP}} \propto \sigma_{0}^{2} / R_{\mathrm{e}} / I_{\mathrm{e}} \propto C_{\mathrm{SF}}{ }^{0.34}$.

Dependence of mass-to-light ratio on the galactic luminosity implied by the scaling relation of the FP requires that $K_{\mathrm{FP}}(\propto M / L)$ should depend on $L$ as $K_{\mathrm{FP}} \propto L^{0.14}$ (e.g., Pahre et al. 1995; Djorgovski et al. 1996). In the present study, the $K_{\mathrm{FP}}$ is found to depend on the $C_{\mathrm{SF}}$ as $K_{\mathrm{FP}} \propto C_{\mathrm{SF}}{ }^{0.34}$. By using the above two dependences of the $K_{\mathrm{FP}}$, we can obtain the result that the $C_{\mathrm{SF}}$ should depend on $L$ as $C_{\mathrm{SF}} \propto L^{0.41}$ for explaining the origin of the FP slope. This expected dependence of $C_{\mathrm{SF}} \propto L^{0.41}$ implies that if more luminous elliptical galaxies are formed by galaxy mergers with more rapid star formation, the slope of the FP can be at least qualitatively explained in the present merger model. This result furthermore demonstrates that although a specific relation between star formation history of galaxy mergers 

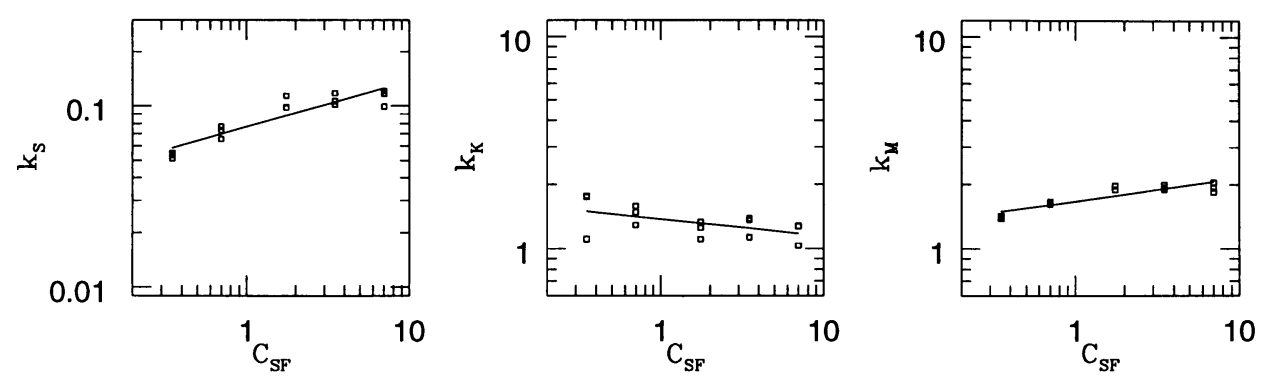

Figure 1. Dependence of nonhomology parameters, $k_{\mathrm{S}}, k_{\mathrm{K}}$, and $k_{\mathrm{M}}$ on $C_{\mathrm{SF}}$. Physical meanings of the three nonhomology parameters are described in the manuscript. The parameter values projected onto $x y, x z$, and $y z$ plane are plotted by open squares for five models with different $C_{\mathrm{SF}}$ in the Model 1 . A solid line in each panel indicates the best fitted line derived by least square fitting procedure for each set of results.

and galactic luminosity is required, the origin of the FP slope can be closely associated with the star formation history of elliptical galaxies formed by dissipative galaxy merging. Although the present merger model is indeed rather idealized and less realistic for real dissipative galaxy mergers with star formation at higher redshift universe, it appears to have succeeded in demonstrating that the origin of the FP can reflect a close physical relation between galactic luminosity and star formation history of merger progenitor galaxies, in particular, the rapidity of star formation of galaxies.

\section{References}

Bekki, K., \& Shioya, Y. 1997, ApJ, 478, L17

Djorgovski, S., \& Davis, M. 1987, ApJ, 313, 59

Djorgovski S., de Carvalho R., \& Han M-S., 1988, in Extragalactic distance scale ed. S. Van den Bergh, and J. P. Christopher, ASP Conf. Ser. Vol. 4, p. 329

Djorgovski, S., Pahre, M. A., \& de Carvalho R. R. 1996, in Fresh Views of Elliptical Galaxies, ed. A. Buzzoni, R. Renzini, and A. Serrano, ASP Conf. Ser. Vol 86, p129

Dressler, A., Lynden-Bell, D., Burstein, D., Davies, R. L., Faber, S. M., Terlevich, R. J., \& Wegner, G. 1987, ApJ, 313, 42

Fall, S. M., \& Efstathiou, G. 1980, MNRAS, 193, 189

Pahre, M. A., Djorgovski, S., \& de Carvalho R. R. 1995, ApJ, 453, L17

Prugniel Ph., \& Simien F. 1996, in Fresh Views of Elliptical Galaxies, ed. A. Buzzoni, A. Renzini, and A. Serrano, ASP Conf. Ser. Vol. 86, p. 151

Renzini, A., Ciotti, L., D'Ercole, A, \& Pellegrini, S. 1993, ApJ, 419, 52

Schmidt, M. 1959, ApJ, 344, 685

Toomre, A., \& Toomre, J. 1972, ApJ, 178,623 